\title{
The Medical Care Continuity (MCC) project. A pilot study of video-assisted home care within the eTEN European Community program. The Italian experience
}

\author{
V. Formica • E. Fossile • R. Pellegrino • M. Fatale • \\ M. Mari • M. Rabuffetti • F. R. Benedetto • G. Visconti • \\ E. Bollero • M. Roselli
}

Received: 10 July 2008 /Accepted: 2 December 2008 / Published online: 17 December 2008

(C) Springer-Verlag 2008

\begin{abstract}
Goals of work This study aimed to use an integrated system (Medical Care Continuity (MCC) system) consisting of computer, video telephone, and a high-definition camera to monitor at home chemotherapy side effects in cancer outpatients.

Patients and methods The system allowed $24 \mathrm{~h} /$ day video consultation with an intermediate medical call center with possible connection to a specialized hospital if necessary. All patients were provided with internationally validated and project-oriented questionnaires exploring patients' health status and opinions on usefulness and complexity of study devices. The content of each call was recorded on a computer database. An approximate estimate of avoided hospital admissions was calculated.

Main results Median duration of experimentation and frequency of patient/doctor contacts were 2.1 months and 4.2 contact per week, respectively. Overall, a $98 \%$ positive opinions on the experimental system was reported at the end of the study by all participants, with a $21 \%$ conversion rate with respect to the opinions gathered at study entry. Changes
\end{abstract}

V. Formica $\cdot$ E. Fossile $\cdot$ R. Pellegrino $\cdot$ M. Roselli $(\triangle)$

Medical Oncology Unit, Internal Medicine Department,

"Tor Vergata" Clinical Center, University of Rome,

Viale Oxford, 81,

00133 Rome, Italy

e-mail: mario.roselli@ptvonline.it

M. Fatale $\cdot$ M. Mari $\cdot$ M. Rabuffetti

AXA Assistance,

Rome, Italy

F. R. Benedetto $\cdot$ G. Visconti $\cdot$ E. Bollero

General Direction, "Tor Vergata" Clinical Center,

University of Rome,

Rome, Italy in patient management after a medical call were made in $32 \%$ of cases. It was calculated that approximately 2.2 per month unnecessary hospital admissions were avoided.

Conclusions The MCC system was well managed by both patients and caregivers. These results show that it has the potential to improve medical assistance by virtue of a constant access to medical advice and reduce unnecessary hospital admissions.

Keywords Video-assisted home care Chemotherapy Medical care continuity

\section{Introduction}

Cancer patients treated in a hospital setting with polychemotherapy regimens, intravenously or orally, may experience several side effects when back home.

In such an eventuality, guidelines provide a number of supportive therapies to be administered (e.g., antiemetic therapy, growth factors, etc.) with no particular need for hospitalization.

Most reported side effects on chemotherapy are nausea/ vomit, mucositis, diarrhea, neutropenia, anemia, thrombocytopenia, hyperpyrexia, hand-foot syndrome (HFS), alopecia, and peripheral neurotoxicity.

For instance, a standard regimen used for metastatic breast cancer containing both anthracyclines and taxanes may cause $\sim 100 \%$ of severe neutropenia $(<1,000$ neutrophils per cubic millimeter) and $30-40 \%$ of febrile neutropenia during the home stay [16].

This requires careful monitoring of body temperature, checking for signs and symptoms of infection, repeated blood test checks, as well as prescription of supportive care drugs (growth factors, antibiotics) outside the hospital. 
Moreover, a growing body of evidence suggests that cancer patients can be effectively treated with several types of oral anticancer drugs and possibly followed with no hospital admission.

For example, capecitabine, an oral pro-drug of chemotherapeutic agent 5-fluorouracil, is widely used for the treatment of colorectal and breast cancers. It may cause relevant toxicity involving skin and mucosa, such as HFS and severe diarrhea $[4,12]$. In a recent study including more than 1,200 patients with metastatic colon cancer, $47 \%$ and $53 \%$ of diarrhea and HFS were reported, respectively [4].

In addition, cancer patients may amplify their perception of discomfort and symptoms while at home and their understandable anxiety may contribute to the increase of unnecessary hospital visits, thus increasing medical expenses.

Cancer patients need a complex clinical, social, and psychological management that can be effective only if care continuity from hospital to home is guaranteed.

A daily visual connection between health care professional and patient may be of great value in reducing patient anxiety, allowing accurate skin toxicity teleassessment and providing a more complete picture of patient status thus avoiding unnecessary outpatient visits.

Furthermore, remote-controlled high-definition camera installed at the patient's home may allow physicians to view, at the hospital site, medical reports from radiological exams, blood tests, etc. that patients keep at home.

Hence, the idea of a research project (the MCC project) to enable physicians to properly evaluate post-chemotherapy side effects with the support of computer and video telephone was developed. In recent years, we have seen extraordinary advances in telecommunication technology and the use of such new technological tools for medical purposes has only recently become a matter of research. Video-assisted medical care is increasingly being used to deliver diagnostic and therapeutic support.

The main objective of the present study was to assess the feasibility of video-assisted home care of cancer patient on chemotherapy and evaluate a possible reduction of unnecessary hospitalizations and hospital visits via a prompt solution of medical problems with the use of a direct patient/physician video link.

\section{Patients and methods}

The study was conducted in two separate phases

\section{Screening phase}

Consecutive patients receiving chemotherapy for solid tumors at the Medical Oncology Unit of Tor Vergata Clinical Center (PTV) between November 2005 and
October 2006 were screened for study eligibility and willingness to participate in the study. Screened patients were classified by tumor primary site, extent of disease, Karnofsky performance status, and type of chemotherapy. No selection for patient screening was made in terms of disease extension (metastatic vs localized), type of chemotherapy (adjuvant vs first-line treatment for metastatic disease, standard chemotherapy vs biological drugs, intravenous vs oral administration), and management issues (general medical conditions, comorbidity, psychological, social, and familial issues).

Data regarding four variables potentially influencing patient decision to participate in the study were analyzed: (1) annual income, (2) education level, (3) traveling distance between patients' homes and PTV, (4) age. Patient subgroups were identified for each variable as follows: high vs medium-low annual income (considering as cutoff 50,000 euros per year), primary school vs high school/ university education, easy access vs difficult access to the hospital (considering a cutoff traveling time of $30 \mathrm{~min}$ ), and $>60$ vs $<60$ years of age.

Acceptance rate for study participation was determined in each patient subgroups.

Patients consenting to the study accepted to have, if available, the MCC platform installed at home (see "MCC platform and architecture" section) and provided a written informed consent. A signed agreement was also obtained from respective general practitioners and caregivers.

\section{Experimental phase}

MCC platform and architecture As the current project was a pilot feasibility study, the experimental equipment was of limited availability and, among patients who consented to the study, five subjects were selected to have one of the five available video stations installed at home for video-assisted MCC between each cycle of chemotherapy. The five patients were selected at investigator discretion as emblematic cases of all treatment types (oral chemotherapy, standard intravenous chemotherapy, and new biological drugs), disease conditions (both metastatic disease and with no evidence of disease on adjuvant chemotherapy), and ages.

The MCC platform consisted of a remote assistance equipment (videophone, webcam, remote control) installed permanently at the hospital, at an intermediate call center, and at the patient's home for the duration of the treatment (Fig. 1). Main aspect was a patient video monitoring which was only used at required times with the patient's agreement. The patient was able to contact at any time the call center, which was in charge of an initial medical assessment. The call center was able to forward the video call to the hospital (PTV) as a specialized medical management was required. Hospital and call center were 


\section{Functional Model}

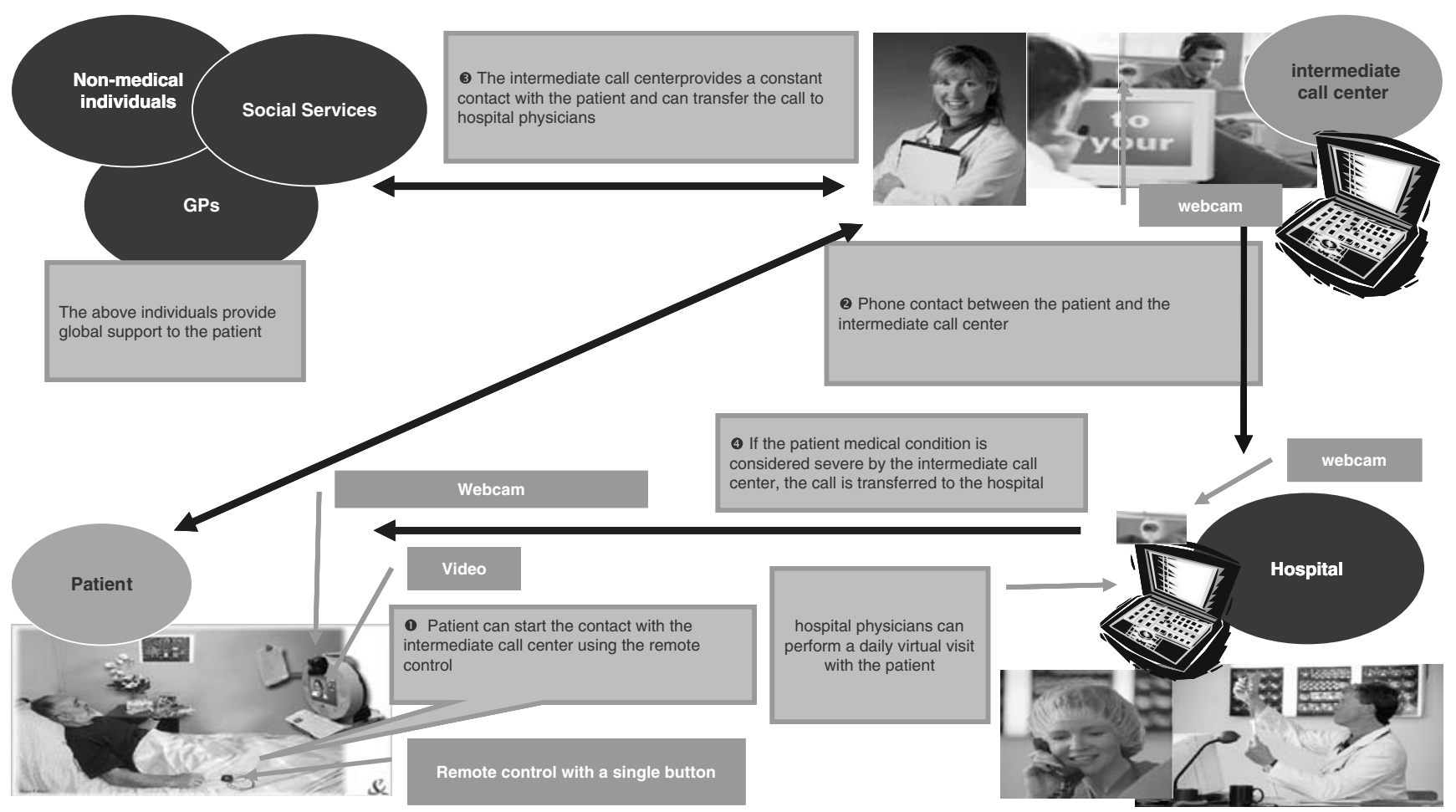

Fig. 1 The Medical Care Continuity $(M C C)$ functional model: The MCC architecture allows the patient to contact at any time $(24 \mathrm{~h} /$ day) the intermediate call center. The call center can forward the video call to the hospital as a specialized medical management is required. GPs: general practitioners

family member, and caregiver opinions on five relevant aspects of the MCC system:

1. Potential improvement of patient health

2. Complexity of MCC technology

3. Perception of videophone at home as a disturbing factor

4. Potential improvement of medical assistance

5. Feeling of protection and reassurance.

The MCC questionnaire was also administered to study investigators in order to explore physicians' points of view.

All questionnaires were to be completed at the beginning and at the end of the experimental phase.

Karnofsky Performance Status [9] was also recorded by investigators at study entry and at each treatment cycle thereafter throughout the study.

All video calls were traced in a unique database and data regarding number of calls a week, reason for video calling (technical vs medical problems), and duration of each video call were analyzed. For each call, detailed notes on topics of conversation, patient general conditions, side effects related to chemotherapy, and physician advices on medical management were also recorded in the database. Number of calls resulting in change of patient medical management (i.e., prescription of new drugs, drug dose modification, medical advices on patient diet and activities of daily living, etc.) were considered an index of MCC property of giving

additional tailor-made questionnaire (MCC projectspecific questionnaire) was designed to explore patient, 
physicians all necessary medical information for a complete decision-making process.

At the end of each call, patients and physicians had to double-blindly answered to the following question: would you have requested a hospital admission or visit if the MCC system had not been available? Number of positive answers were considered a rough estimate of avoided unnecessary hospital admissions.

\section{Statistical analyses}

All rate differences between patient subgroups were analyzed using the chi-squared test. Statistical significance was considered for $p<0.05$.

\section{Results}

\section{Screening phase}

In the screening phase, 80 consecutive patients receiving chemotherapy for solid tumors at the PTV between November 2005 and October 2006 were approached. An attempt to include a wide variety of cancer types and patients was made in order to render the screening group as representative as possible of the actual cancer patient profile referred to our institution.

Participants had different cancer disease conditions (metastatic vs radically resected patients) and various general management issues (general medical condition, comorbidity, psychological, social, and familial issues). Caregiver availability was also taken into consideration.

Among the 80 screened patients, tumor type and extent of disease were distributed as follows: breast cancer 17 patients (eight on adjuvant treatment, nine metastatic), gastrointestinal cancer 34 (nine on adjuvant treatment, 25 metastatic), lung cancer 15 (two on adjuvant treatment, 13 metastatic), other cancer types 14 .

Screened patients came from a wide catchment area with patients' houses being located all over the urban area of Rome. A specific analysis was generated to investigate whether the annual income as a measure of the socioeconomic status, education level, accessibility to the hospital, or the age (considered as a parameter influencing the ability of patients of using more advanced technological tools) could affect patient willingness to accept and participate in the study. As shown in Table 1, there were no statistically significant differences in the acceptance rate for the study when patients were divided between high annual income and mid-low annual income and between low education level and high education level. No differences were also seen between patients with easy accessibility to the hospital and difficult accessibility to the hospital and patients over and under 60 years old, with an overall high acceptance rate, ranging from $65 \%$ to $79 \%$.

\section{Experimental phase}

\section{Patient characteristics}

Based on the availability of only five video stations to be installed, five cancer patients treated with chemotherapy were enrolled for the experimental phase between November 2006 and March 2007. Each patient was identified by a number from 01 to 05 . Three females and two males entered into the study with a median age of 69 years (4476). All the three females suffered from breast cancer; the two males had colon cancer. Three patients were receiving chemotherapy for metastatic disease (two breast, one colon cancer); the other two patients were having adjuvant chemotherapy with no evidence of disease (see Table 2 for patient characteristics).

Table 1 Differences in acceptance rate among patient subgroups

\begin{tabular}{|c|c|c|c|c|}
\hline & & Acceptance rate & Chi-squared $p$ value & \\
\hline \multirow[t]{2}{*}{ Annual income } & High & $79 \%$ & 0.156 & ns \\
\hline & Medium-low & $65 \%$ & & \\
\hline \multirow[t]{2}{*}{ Education level } & Primary school & $69 \%$ & 0.467 & ns \\
\hline & High school/university & $76 \%$ & & \\
\hline \multirow[t]{2}{*}{ Distance between patients' homes and hospital } & Close & $69 \%$ & 0.523 & ns \\
\hline & Far & $76 \%$ & & \\
\hline \multirow[t]{2}{*}{ Age } & $>60$ years old & $74 \%$ & 0.678 & ns \\
\hline & $<60$ years old & $70 \%$ & & \\
\hline
\end{tabular}

Differences in acceptance rate for study participation among patient subgroups. Data regarding four variables potentially influencing patient decision to participate in the study were analyzed. Patient subgroups were identified as follows: high vs medium-low annual income (considering as cutoff 50,000 euros per year), primary school vs high school/university education, easy access vs difficult access to the hospital (considering a cutoff traveling time of $30 \mathrm{~min}$ ), $>60 \mathrm{vs}<60$ years of age

$n s$ not significant 
Table 2 Study patient characteristics

\begin{tabular}{llllllr}
\hline Patient & Age at study entry & Primary tumor & Disease status & Sex & Type of chemotherapy & Time from initial cancer diagnosis (years) \\
\hline 01 & 76 & Breast & M+ & F & Trastuzumab (iv) & 13.5 \\
02 & 44 & Breast & M+ & F & Capecitabine (oral) & 3.7 \\
03 & 70 & Breast & NED & F & CMF (iv) & 0.6 \\
04 & 69 & Colon & NED & M & FOLF (iv) & 0.6 \\
05 & 64 & Colon & M+ & M & FOLFIRI (iv) & 2.3 \\
Median & 69 & & & &
\end{tabular}

Study patients' characteristics. Each patient is identified by a number from 01 to 05

$M+$ metastatic disease, $N E D$ no evidence of disease, $M$ male, $F$ female, $C M F$ cyclophosphamide-methotrexate-fluorouracil regimen, $F O L F I R I$ folinate-fluorouracil-irinotecan regimen, $F O L F$ folinate-fluorouracil regimen, iv treatment administered intravenously, oral treatment administered orally

Included subjects had different ages and both patients with metastatic disease and patients with no evidence of disease on adjuvant chemotherapy were recruited. All types of antineoplastic therapies were represented, namely, oral chemotherapy, standard intravenous chemotherapy, and new biological drugs. Median interval time between initial disease diagnosis and study entry was considered as relevant factor determining psychological acceptance of disease status. The median interval time between diagnosis and study entry was 2.3 years $(0.6-13.5)$.

\section{Video calls}

Frequency of patient/doctor video contacts was determined by calculating the total number of contacts per patient/total weeks of study period.

Median duration of the experimental phase among the patients was 2.1 months (1.7-4.1). Median frequency of patient/doctor video contacts was 4.2 contacts per week (2.1-8.4; Fig. 2).

Average proportions of contacts due to technical support and medical reasons were $34 \%(9 \times 61 \%)$ and $66 \%(39-$ $85 \%)$, respectively. There was an approximately fixed number of video calls made for technical reasons that mainly occurred in the first period of study to set up the system. As the time on study passes by (as it was for the first enrolled patient, patient 01 , with over 4 months of study), calls due to medical reasons become the prevalent type of calls.

\section{Questionnaire pack}

Considering on the whole the five patients, relative caregivers (which were predominantly patients' partners), and the three hospital physicians involved in the study, a score of $98 \%$ of favorable answers to the specific questionnaire on MCC system characteristics was recorded at the end of the study (Fig. 3). Nearly all of them reported positive opinions on patient health improvement by MCC, simplicity of MCC technology, not-disturbing presence of videophone at home, protective and reassuring role of MCC, and amelioration in medical assistance, while before starting the study a $77 \%$ score of favorable answers was documented.

Main changes of opinion were reported for MCC ability of improving health condition, simplicity of MCC technology,
Fig. 2 Data on call frequency, reasons for calling, and period on study for each single patient. Each patient is identified by a number from 01 to $05 . p$ : patient; $n$ : number of calls

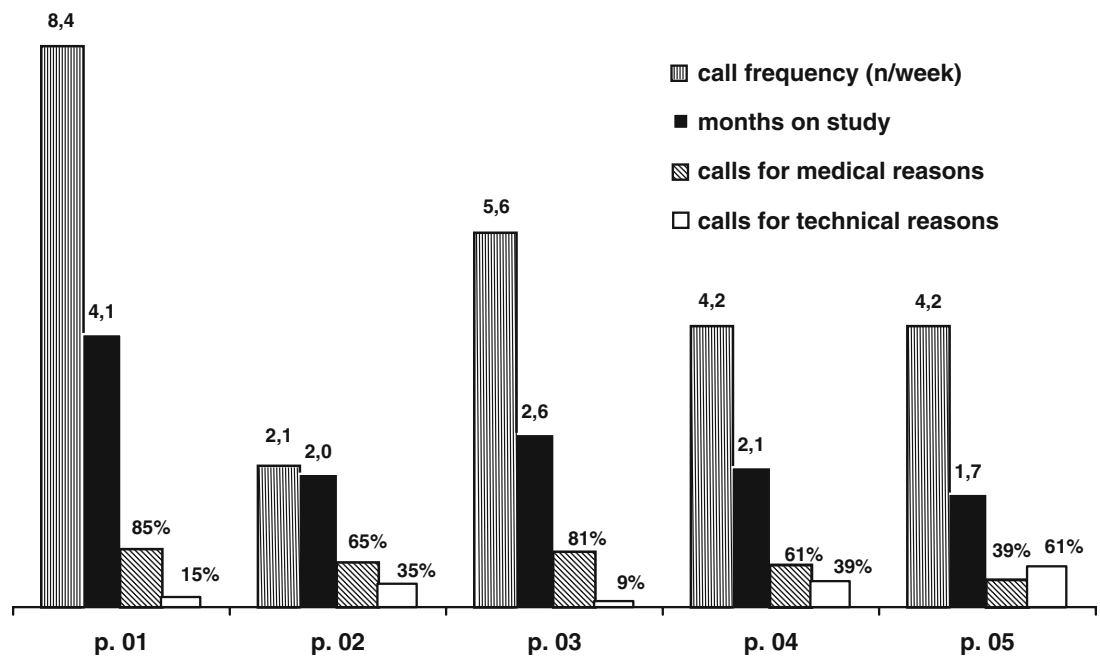


a

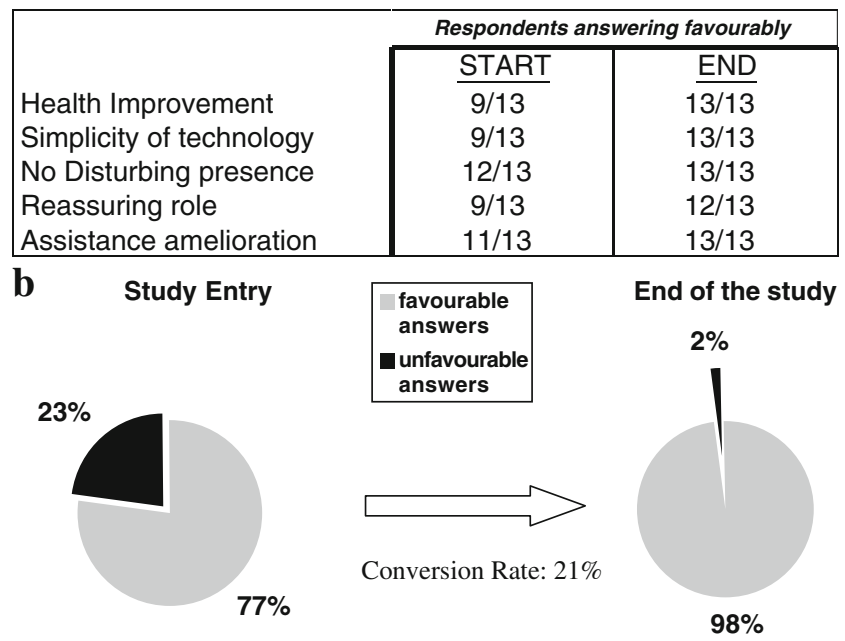

Fig. 3 Results from a specific questionnaire exploring patients' (n.5), respective caregivers' (n.5), and hospital physicians' (n.3) opinions on five relevant aspects of the MCC system: (1) potential improvement of patient health, (2) complexity of MCC technology, (3) perception of videophone at home as a disturbing factor, (4) potential improvement of medical assistance, and (5) feeling of protection and reassurance. The questionnaire was to be completed at the beginning and at the end of the experimental phase. a Number of respondents (out of the total of 13) answering favorably to each of the five specific questions on MCC system characteristics at the beginning (START) and the end $(E N D)$ of the study. b Overall proportions among all respondents of favorable and unfavorable opinions at the beginning and the end of the study. Overall, $21 \%$ of opinions were converted from unfavorable to favorable after the study was completed

and MCC ability of improving medical assistance (overall $21 \%$ conversion rate from unfavorable to favorable answers Fig. 3).

According to HAD results (Fig. 4a), neither anxiety nor depression at significant levels were documented before study commencement for three out of five patients, while mild to moderate anxiety and depression were reported for two patients, with no significant changes on the whole before and after the experimental phase (Fig. 4a). No significant differences between before and after MCC intervention were also documented in Karnofsky Performance Status (Fig. 4a), SF-36 test (Fig. 4b), and KATZ index (data not shown).

\section{Decision-making process}

When data on detailed information of video-call contents were analyzed, it was revealed that, on average, $32 \%$ (range $20-47 \%$ ) of calls resulted in the decision of changing patient medical management taken by hospital physicians. This value was considered indicative of a good quality and informativeness of the data collecting via the MCC device that allowed a complete decision-making process (Fig. 5). Types of medical management change were clearly distinct when metastatic- and adjuvant-chemotherapy-receiving patients were considered separately. In fact, for metastatic patients, $35 \%$ of medical contacts resulted in oral drug dose adjustment (mainly pain killers and oral chemotherapy), while $22 \%$ of medical contacts with patients on adjuvant treatment were devoted to anxiety relief and management of post-surgical complications (i.e., arm lymphedema in breast cancer patients; data not shown).

Figure 6 represents the number of occasions per month in which, after a call, there would have been a request of hospital admission/visit if MCC device had not been available, according to both patients' and hospital physicians' perspective. This parameter was considered as a rough estimate of avoided unnecessary hospital admissions/ visits. Considering physician perspective as the most reliable, an average of 2.2 hospital accesses per month (range 3-1.5) were virtually avoided by managing the patient at home using the video platform, with a real impact in terms of health system cost saving.

\section{Discussion}

Major interest is currently being shown by health care systems and medical professionals about the potential use of new telecommunications technologies to support cancer care. Encouraging results have been achieved in the fields of oncological teleconsultations for both patients and professionals $[10,11,14]$ and telementoring during surgical procedures for cancer disease $[3,15]$. Still little data though are available on telecare for cancer patients on chemotherapy experiencing side effects from the treatment while at home. In the present study, we demonstrated that videoassisted home care is feasible and reassuring and may potentially limit unnecessary hospital admissions.

Request for video-assisted virtual home visit may be estimated as very high among patients suffering from cancer with respect to non-oncological patients. In a study by Hebert et al., a retrospective chart review on 354 palliative care visits of patients with either oncological or non-oncological disease care was conducted. A professional nurse judged whether video visits could have been carried out. Approximately $43 \%$ of the visits were considered interchangeable with video visits. Cancer patients were more likely to be considered suitable for video visits, which suggests that type of disease, rather than diagnosis of "palliative," may be more influential for appropriateness of videophone use [7].

However, this approach might have some limits due to the fact that patients more able to use electronic devices (computer) might be more easily recruited determining a discriminating factor in the enrollment of new patients into the program. 
Fig. 4 Results from internationally validated tests. Each patient is identified by a number from 01 to 05. p.: patient. a Results from Hospital Anxiety and Depression and Karnofsky Performance Status $(P S)$ tests at the beginning (START) and at the end $(E N D)$ of the study. $<8$ : no anxiety/depression at significant levels; $8-11$ : mild to moderate anxiety/depression; $>11$ : significant levels of anxiety/depression. For the legend of

Karnofsky PS values, see [9]. b SF-36 test. Black columns: SF36 results at study entry, white columns: SF-36 results at the end of the study. For the legend of SF-36 test values, see references [18, 19] a

\begin{tabular}{|l|l|l|l|l|l|l|}
\hline & \multicolumn{3}{l}{ Anxiety } & \multicolumn{2}{l|}{ Depression } & \multicolumn{2}{l|}{ Karnofsky PS } \\
\hline & START & END & START & END & START & END \\
\hline p. 01 & $8-11$ & $>11$ & $8-11$ & $8-11$ & 90 & 80 \\
\hline p. 02 & $8-11$ & $8-11$ & $<8$ & $<8$ & 100 & 100 \\
\hline p. 03 & $<8$ & $<8$ & $<8$ & $<8$ & 100 & 100 \\
\hline p. 04 & $<8$ & $<8$ & $<8$ & $<8$ & 90 & 90 \\
\hline p. 05 & $<8$ & $<8$ & $<8$ & $<8$ & 100 & 90 \\
\hline
\end{tabular}

b

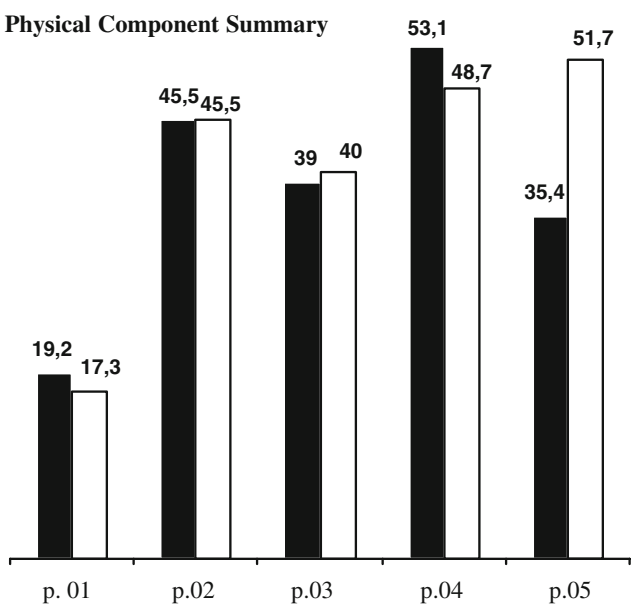

Mental Component Summary

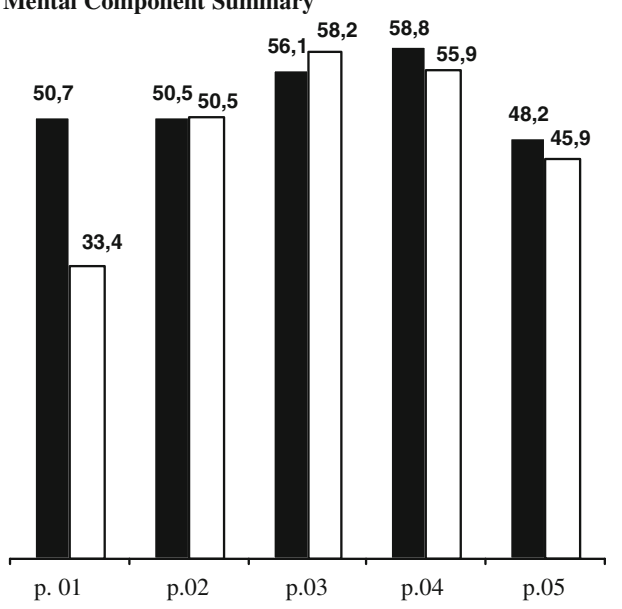

In our study, the MCC equipment was well managed by both patients and caregivers; the main positive change in patient and family member perspective was the perception that MCC may substantially improve medical assistance by virtue of a constant ( $24 \mathrm{~h} /$ day) access to medical advice with $21 \%$ of opinions changing from unfavorable at study entry to favorable at the end of the study. Study design was appropriate with a high acceptance rate for study participation among all screened cancer patients regardless of their socio-economic status, education level, and easy access to the hospital, suggesting that the ability to interface with electronic devices did not depend on these variables.

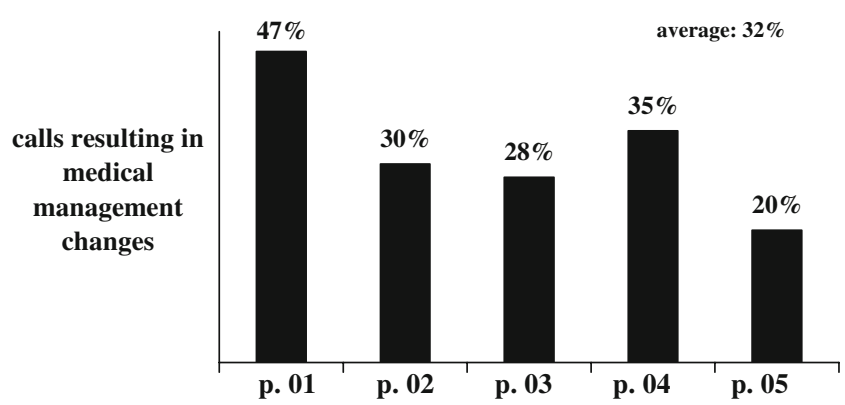

Fig. 5 Percentage of calls for each study patient resulting in the decision of changing patient medical management taken by hospital physicians. Each patient is identified by a number from 01 to $05 . p$.: patient. Values were considered indicative of the quality and informativeness of the data collecting via the MCC system
Even if an anxiety-level reduction would have been expected, no changes in HAD scores were documented for all participants.

Although these data refer to a limited number of patients and a relatively short follow-up, preliminary results are encouraging. Assuming that physician's point of view is more reliable in determining the need for hospital visits, it

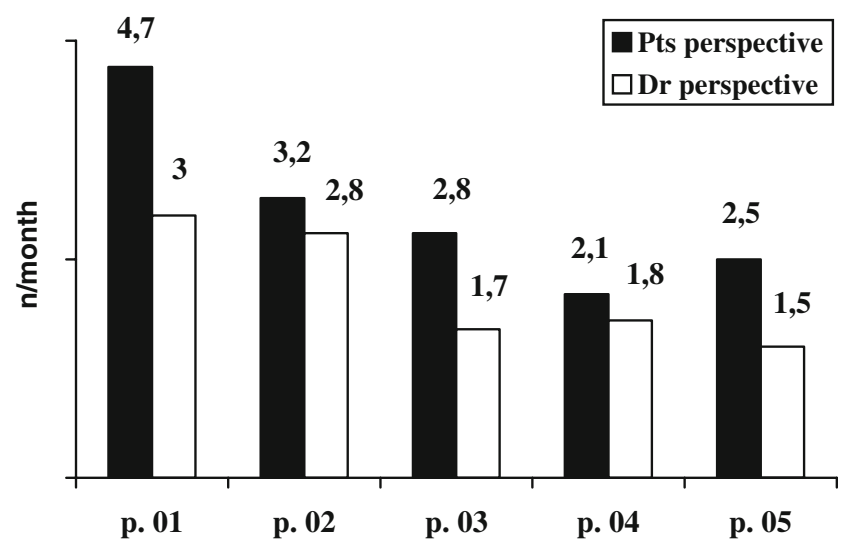

Fig. 6 Number of occasions per month in which, after a call, there would have been a request of hospital admission/visit if MCC system had not been available. Black columns: occasions in which the requests would have come from the patient (Pts perspective), white columns: occasions in which the requests would have come from hospital physicians ( $D r$ perspective). Ro: roman patient (used to generate patient code). $p .:$ patient 
can be estimated that a rate of two to four hospital accesses a month can be avoided by managing the patient at home using the video platform, with a real impact in terms of health system cost saving.

Our results are in keeping with data published by Chumbler et al. [5] that showed how home telehealth technologies may lower clinic visits, bed days of care for hospitalization, and number of hospitalizations among cancer patients undergoing chemotherapy as compared to standard care.

Further experimentations to test such an innovative technology are recommended, especially considering its potential usefulness in following cancer patients on treatment with the emerging oral targeted therapies.

Moreover, a good quality of internet connection and a high definition of screen images cannot be guaranteed everywhere in urban as well as rural areas and with all telephone companies. The experimental equipment still needs in fact improvements from a technical point of view.

Another concerning aspect could be that a constant "video connection" with patient personal lives at home can be regarded as a too-intrusive approach for patients as well as for their relatives. However, data from administered questionnaires have shown that in our study patients and respective caregivers appreciate the reassuring value of having a 24-h-available medical advice via MCC connection with no concern for privacy invasion.

An open question would also remain. The most favorable advantage of MCC project, i.e., the economical gain of reducing unnecessary hospitalizations, must be weighted against the expenses of a highly sophisticated technologies that have not yet a large-scale production and an easy access to the general public.

The present research is a pilot study and it was not possible to reach a larger sample size. Further multicenter studies are required to improve expertise in the equipment use and confirm these results.

\section{References}

1. Berard RM (2001) Depression and anxiety in oncology the psychiatrist's perspective. J Clin Psychiatry 62:58-61

2. Bjelland I, Dahl AA, Haug TT, Neckelmann D (2002) The validity of the hospital anxiety and depression scale. An updated literature review. J Psychosom Res 52:69-77. doi:10.1016/ S0022-3999(01)00296-3

3. Camara JG, Zabala RR, Henson RD, Senft SH (2000) Teleophthalmology: the use of real-time telementoring to remove an orbital tumor. Ophthalmology 107:1468-1471. doi:10.1016/ S0161-6420(00)00211-6
4. Cassidy J, Twelves C, Van Cutsem E, Hoff P, Bajetta E, Boyer M et al (2002) Capecitabine Colorectal Cancer Study Group: a favorable safety profile compared with intravenous 5-fluorouracil/ leucovorin. Ann Oncol 13:566-575. doi:10.1093/annonc/mdf089

5. Chumbler NR, Kobb R, Harris L, Richardson LC, Darkins A, Sberna $M$ et al (2007) Healthcare utilization among veterans undergoing chemotherapy: the impact of a cancer care coordination/home-telehealth program. J Ambul Care Manage 30:308-317

6. Dowd S, Davidhizar R (1999) Opening up to the Katz index. Elder Care 11:9-12

7. Hebert MA, Paquin MJ, Whitten L, Cai P (2007) Analysis of the suitability of "video-visits" for palliative home care: implications for practice. J Telemed Telecare 13:74-78. doi:10.1258/ 135763307780096203

8. Herrmann C (1997) International experiences with the hospital anxiety and depression scale - a review of validation data and clinical results. J Psychosom Res 42:17-41. doi:10.1016/S00223999(96)00216-4

9. Karnofsky DA, Burchenal JH (1949) The clinical evaluation of chemotherapeutic agents in cancer. In: Macleod CM (ed) Evaluation of chemotherapeutic agents. Columbia University Press, New York, pp 199-205

10. Kunkler IH, Prescott RJ, Lee RJ, Brebner JA, Cairns JA, Fielding RG, Bowman A, Neades G, Walls AD, Chetty U, Dixon JM, Smith ME, Gardner TW, Macnab M, Swann S, Maclean (2007) TELEMAM: a cluster randomised trial to assess the use of telemedicine in multi-disciplinary breast cancer decision making. Eur J Cancer 43:2506-2514. doi:10.1016/j.ejca.2007.08.026

11. Larcher B, Arisi E, Berloffa F, Demichelis F, Eccher C, Galligioni E et al (2003) Analysis of user-satisfaction with the use of a teleconsultation system in oncology. Med Inform Internet Med 28:73-84. doi:10.1080/14639230310000600470

12. Lassere Y, Hoff P (2004) Management of hand-foot syndrome in patients treated with capecitabine (Xeloda). Eur J Oncol Nurs 8: S31-S40. doi:10.1016/j.ejon.2004.06.007

13. Lloyd-Williams M, Spiller J, Ward J (2003) Which depression screening tools should be used in palliative care. Palliat Med 17:40-43. doi:10.1191/0269216303pm664oa

14. Mair F, Whitten P, May C, Doolittle GC (2000) Patients' perceptions of a telemedicine specialty clinic. J Telemed Telecare 6:36-40. doi:10.1258/1357633001933925

15. Purkable TL, Bauer JJ (1999) A telementored trans-rectal ultrasound guided prostate biopsy. Stud Health Technol Inform 62:275-277

16. Smith TJ, Khatcheressian J, Lyman GH, Ozer H, Armitage JO, Balducci L et al (2006) 2006 update of recommendations for the use of white blood cell growth factors: an evidence-based clinical practice guideline. J Clin Oncol 24:3187-3205. doi:10.1200/ JCO.2006.06.4451

17. Snaith RP (2003) The hospital anxiety and depression scale. Health Qual Life Outcomes 1:29. doi:10.1186/1477-7525-1-29

18. Ware JE Jr, Gandek B (1998) Overview of the SF-36 health survey and the international quality of life assessment (IQOLA) project. J Clin Epidemiol 51:903-912. doi:10.1016/S0895-4356 (98)00081-X

19. Ware JE Jr, Sherbourne CD (1992) The MOS 36-item short-form health survey (SF-36). I. Conceptual framework and item selection. Med Care 30:473-483. doi:10.1097/00005650199206000-00002

20. Zigmond AS, Snaith RP (1983) The hospital anxiety and depression scale. Acta Psychiatr Scand 67:361-370. doi:10.1111/ j.1600-0447.1983.tb09716.x 